\title{
Characterization of Spirooxazine and Spiropyran Hosted in Poly(Methyl Methacrylate) for Germicidal UV Source Indicator Application
}

\author{
Julien Bonefacino ${ }^{1 *}$, Ming-Leung Vincent Tse ${ }^{1}$, Chi-Fung Jeff Pun ${ }^{1}$, Xin Cheng ${ }^{1}$, \\ Wing Kin Edward Chan ${ }^{1}$, Arjen Boersma², Hwa-Yaw Tam ${ }^{2}$ \\ ${ }^{1}$ Photonics Research Center, Electrical Engineering Department, The Hong Kong Polytechnic University, Hong Kong, China \\ ${ }^{2}$ Department of Responsive Materials \& Coating, Netherlands Organisation for Applied Scientific Research (TNO), \\ Eindhoven, The Netherlands \\ Email: ${ }^{*}$ julien.bonefacino@gmail.com, arjen.boersma@tno.nl
}

Received June 20, 2013; revised July 22, 2013; accepted August 24, 2013

Copyright (C) 2013 Julien Bonefacino et al. This is an open access article distributed under the Creative Commons Attribution License, which permits unrestricted use, distribution, and reproduction in any medium, provided the original work is properly cited.

\begin{abstract}
A characterization of photophysical properties of 1,3-dihydro-1,3,3-trimethylspiro[2H-indole-2,3'-[3H]naphtha[2,1-b] [1,4] oxazine] (spirooxazine) and 1',3'-dihydro-1',3',3'-trimethyl-6-nitrospiro[2H-1-benzopyran-2,2'-(2H)-indole] (spiropyran) compounds hosted in poly(methyl methacrylate) (PMMA) was carried out. The parameters which influenced the photochromic behavior of both compounds were investigated. After ultraviolet (UV) irradiation, it was demonstrated that the temperature was the dominant factor which influenced the deactivation of both materials. For spiropyran, it was demonstrated that the process could be photo-induced with a certain wavelength which we have specified in this manuscript. It was also found that X-Ray did not affect the photo physical properties of spirooxazine and spiropyran. Finally, both materials could be used as low-cost germicidal UV indicators.
\end{abstract}

Keywords: UV Indicator; Water Disinfection; Photochromism; Polymer

\section{Introduction}

Photochromism is defined as the reversible transformation of a chemical specie between two forms having different absorption spectra by photoirradiation [1]. For the last two decades, photochromic compounds have been intensively studied [2-11] for many applications such as optical switch [12], optical memory [13], ophthalmic lens and light filters [7]. This manuscript concentrates on the photophysical properties of 1,3-dihydro-1,3,3-trimethylspiro[2H-indole-2,3'-[3H]naphth[2,1-b][1,4]oxazine] and 1',3'-dihydro-1',3',3'-trimethyl-6-nitro-spiro[2H-1-benzopyran-2,2'-(2H)-indole] also called spirooxazine and spiropyran respectively. They are organic compounds with a closed ring form (Figure 1), which change into an open ring form called merocyanine under UV exposure. Spirooxazine is colorless in its closed ring form and becomes blue [12] under UV irradiation (open ring form), and spiropyran is pale yellow in its closed ring form and becomes dark purple [14] in its open ring form (Figure 2).

\footnotetext{
"Corresponding author.
}

According to literatures, these transformations are reversible under visible illumination and/or thermal stimulation $[15,16]$. Spirooxazine is considered as a promising material to act as a reliable sensor due to fast response time with good photo-fatigue resistance [12]. Previous works had focused on the liquid form of spirooxazine and spiropyran, when studying the photochromic reaction induced by UV irradiation [2].

Recent works had focused on spiropyran and spirooxazine to be used to tune a photonic crystal cavity [17] and as dosimeter [18]. Nanomedecine application also has been reported and it has been showed that spiropyran's photochromic properties could be used for ondemand drug delivery [19]. To our knowledge, this is the first time to characterize these chemical species hosted in PMMA matrix. Here, we studied the parameters which influence their deactivation.

The growing demand for drinkable water has induced researchers to find novel methods to treat water efficiently at a low cost. This is particularly true for underdeveloped countries where sources of drinkable water 
(a)

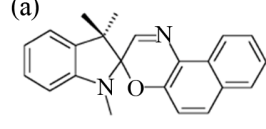

Closed Form

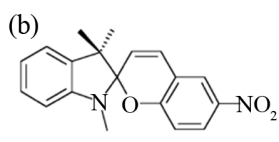

Closed Form
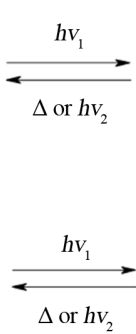

or $h v_{2}$

Figure 1. Chemical structure of spirooxazine and (b) spiropyran

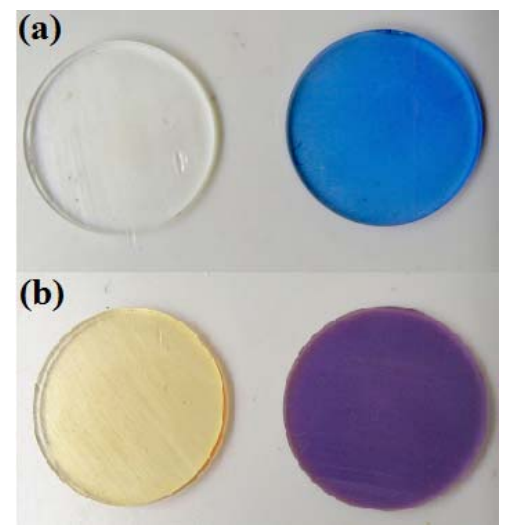

Figure 2. (a) Spirooxazine and (b) spiropyran before (left) and after (right) UV irradiation.

are low and their accesses are limited. A simple method is to use UV-C germicidal wavelength $(\lambda=253.7 \mathrm{~nm})[5$, $20]$ to treat water. This method leads to high annihilation rate of bacteria [21,22], high reliability [6], ease of use and can be done cheaply. These systems inactivate the pathogenic microorganisms that could be responsible of diseases through contamination of water. These systems generate a minimum UV-C dose of $40 \mathrm{~mJ} / \mathrm{cm}^{2}$, and are frequently used in underdeveloped countries for treatment of high quantity of water at reasonable cost ( 2 cents of US dollar per metric ton of water) [10]. In order to detect the amount of UV dose needed to kill bacteria, the system embedded an optoelectronic sensor. The sensor can be expensive, requiring power supply and specific knowledge for maintenance. Therefore, there is a need to develop a low-cost, reliable, user friendly and easy to maintain UV indicator. Spiropyran and spirooxazine are good candidates to act as the required UV indicator due to their photochromism and thermo-optical properties. Instead of optoelectronic sensor, discs of spirooxazine or spiropyran could be used. The discs simply change color when the required UV dose has been reached. Also, other studies have been carried out on germicidal UV sensor using optical fiber $[9,20]$. The objective of our research is to use both spirooxazine and spiropyran as germicidal UV wavelength indicator. In this paper, photochromism and thermo-optic properties characterization of both compounds in PMMA are reported.

\section{Materials and Methods}

\subsection{Materials}

In all the experiments done, herein, spirooxazine and spiropyran with concentration of either $1 \%$ or $3 \%$ were used. Chemicals have been purchased from Sigma-Aldrich. To facilitate the experiments and the intended applications, the compounds were added into a polymethylmethacrylate (PMMA) solution and cured to obtain solid samples. For the sample preparation containing either $1 \%(0.1886 \mathrm{~g})$ or $3 \%(0.5658 \mathrm{~g})$ of photochromic species, the PMMA solutions were made of $20 \mathrm{~mL}$ of Methyl methacrylate (MMA), $0.0225 \mathrm{~g}$ of Benzoyl peroxide (LP) and $112.8 \mu \mathrm{L}$ of 1-Dodecanethiol (DT). MMA was the monomer, LP was used as an initiator to start the polymerization of the monomer and DT introduced a chain transfer to control the molecular weight and to end the polymerization process. After curing, cylindrical optical solids with diameter of $1.3 \mathrm{~cm}$ were fabricated, which were cut into discs of $1.1 \mathrm{~mm}$ thickness (Figure 2) for testing.

\subsection{Photo Physical Characterization}

We used a spectrophotometer (Varian Cary 300 Conc) with wavelength range of $\lambda=200 \mathrm{~nm}$ to $\lambda=800 \mathrm{~nm}$ to measure the absorbance spectra of both materials before and after UV laser exposure. The intensity was set by optics and attenuators to $8 \mathrm{~mW} / \mathrm{cm}^{2}$ and the time of exposure was set to 5 minutes for a total dose of $2.4 \mathrm{~J} / \mathrm{cm}^{2}$. Activation of both chemical species was achieved by illuminating the samples with a $325 \mathrm{~nm}$ UV laser. These compounds can be deactivated and return to their stable state (closed ring form) after exposure to visible light and/or thermally. The influence of temperature on the recovery time of both compounds has been studied. According to the spectra recorded with the spectrophotometer, the wavelength selection also has an effect on the recovery of the open ring forms. For the recovery part, we used a DPSS green laser $(\lambda=532 \mathrm{~nm})$ and a laser pointer FU650AD100-GC18 which emit at $\lambda=650 \mathrm{~nm}$. To avoid additional thermal effect, we set the dose rate of both lasers at a low intensity $(1 \mathrm{~mW})$ for a course of 8 minutes.

\section{Results and Discussion}

\subsection{Absorbance Measurements}

The absorbance spectra of spirooxazine and spiropyran were recorded before $325 \mathrm{~nm}$ exposure. They showed that both compounds had a high absorbance in the UV range (Figures 3(a) and (b)), particularly in the UV-B ( $\lambda$ 
$=280 \mathrm{~nm}$ to $\lambda=315 \mathrm{~nm})$ and second half of UV-C $(\lambda=$ $153 \mathrm{~nm}$ to $\lambda=280 \mathrm{~nm}$ ) band. It is obvious from Figure 3(a) that when the concentration of spirooxazine in the PMMA increased, the UV absorbance value and bandwidth increased, and the absorbance spectrum became sharper. Note that the curves between the 2 vertical lines (from 300 to $360 \mathrm{~nm}$ ) of Figures 3 and $\mathbf{4}$ may not represent the actual measurements as the spectrophotometer switched sources around $350 \mathrm{~nm}$. Thus, the results obtained in this range were not accurate. The absorbance spectrum in the visible was nearly the same for spirooxazine with different concentration. Moreover, the absorbance at germicidal wavelength $(\lambda=254 \mathrm{~nm})$ was high for this compound with values of 2.95 and 3.31 for concentration of $1 \%$ and $3 \%$, respectively.

The measurement for spiropyran (Figure 3(b)) before $325 \mathrm{~nm}$ exposure showed that for concentration of $1 \%$, this photochromic compound was more sensitive than spirooxazine (1\% and 3\%) as its absorbance was higher, which led to a better conversion from closed ring form to open ring form. Also, note that the absorbance at $\lambda=254$ $\mathrm{nm}$ was 3.66 which was the highest we recorded. The results obtained with concentration of $3 \%$ showed that the absorbance of spiropyran was lower than that of concentration of $1 \%$. The closed ring form of this material can even changed into the open ring form by ambient light illumination, led to a decrease of the overall UV absorbance. From Figure 3(b), an absorbance peak with
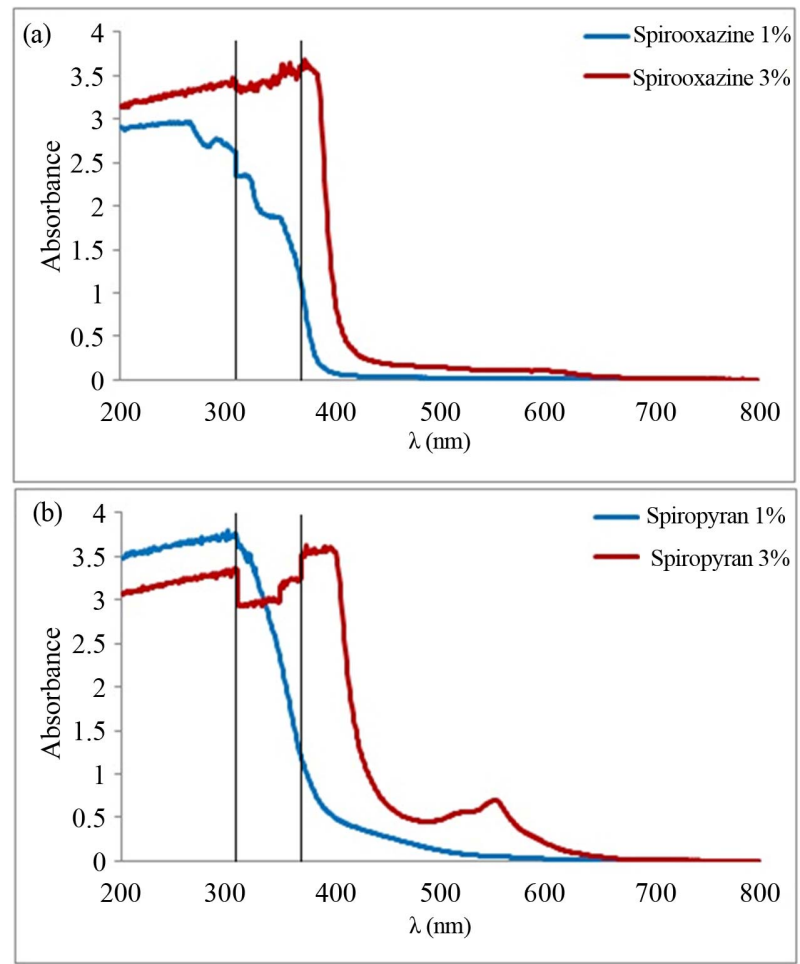

Figure 3. (a) Absorbance of spirooxazine and (b) spiropyran before UV irradiation.
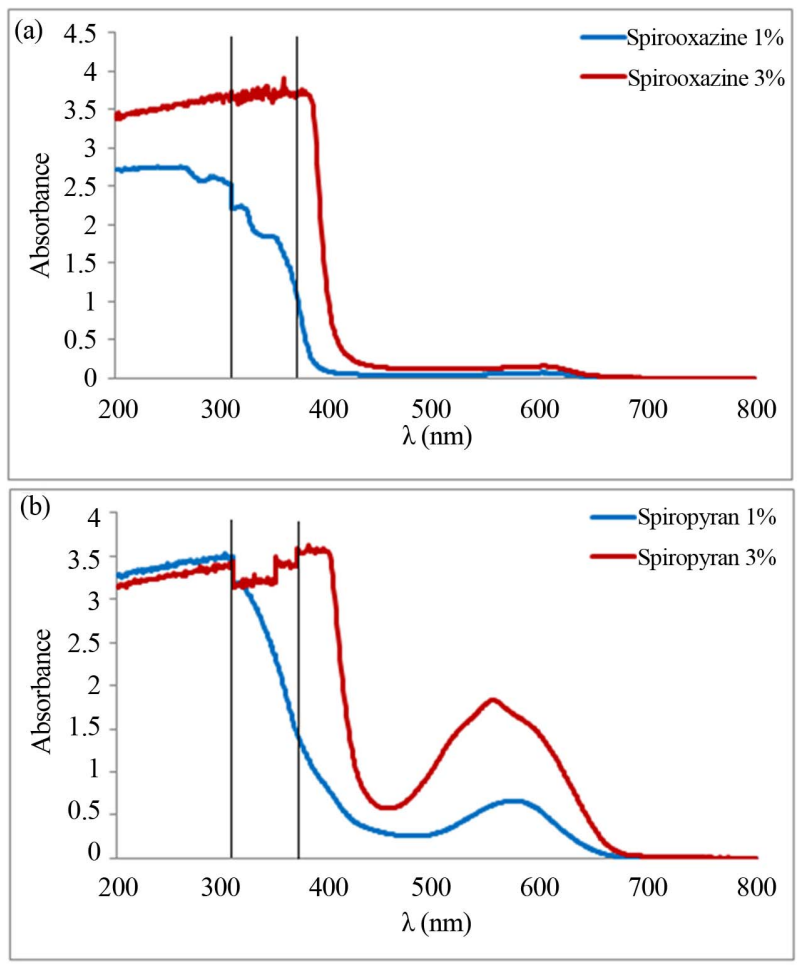

Figure 4. (a) Absorbance of spirooxazine and (b) spiropyran after UV irradiation.

a maximum of 0.7 at $\lambda=550 \mathrm{~nm}$ could be observed, which was further evidence for the explanation given above as the results recorded after a 5 minutes $325 \mathrm{~nm}$ exposure provided similar effect.

Next, the compounds were irradiated with a $325 \mathrm{~nm}$ laser, and the activation of both compounds led to a change in color for both samples. Fluorescence at $\lambda=450$ $\mathrm{nm}$ for the spirooxazine and $\lambda=655 \mathrm{~nm}$ for the spiropyran was observed. The absorbance spectrum (Figures 4(a) and (b)) after activation under $325 \mathrm{~nm}$ for 5 minutes were recorded. The absorbance in the UV band of both materials with a concentration of $1 \%$ and $3 \%$ was approximately the same as that recorded before irradiation (Figure 3). From Figure 4(a), the absorbance spectrum of spirooxazine after laser exposure was similar to the one recorded before (Figure 3(a)). The absorbance's values and bandwidth in the UV were similar, with an increase for concentration of 3\%. Moreover, the absorbance in the visible range was almost the same with a low value which contradicted previous observations [12]. The most interesting results have been obtained for the sample containing spiropyran (Figure 4(b)). The spectra before (Figure 3(b)) and after $325 \mathrm{~nm}$ irradiation (Figure 4(b)) were similar in the UV range, however, the materials exhibited a significant increase in absorption in the visible region. This was due to the activation of the material from closed ring form to merocyanine form under UV irradiation [3,15,21]. 
For concentration of $1 \%$, the peak was center at $\lambda=$ $572 \mathrm{~nm}$ with an absorbance of 0.66 , and it ranged from $\sim 480 \mathrm{~nm}$ to $630 \mathrm{~nm}$, which was consistent with the previous spectra observed [4]. For concentration of $3 \%$ of spiropyran, it was observed that after $325 \mathrm{~nm}$ irradiation, an increase of the absorbance value and bandwidth of the visible peak, centered at $\lambda=553 \mathrm{~nm}$ with a value of 1.83 , and a bandwidth ranged from $460 \mathrm{~nm}$ to $637 \mathrm{~nm}$. Furthermore, the peak shifted to the shorter wavelength when the concentration increased, with a measured shift of $19 \mathrm{~nm}$.

It could be concluded that both spirooxazine and spiropyran were very sensitive to $\mathrm{UV}$, and after irradiation, only spiropyran exhibited absorbance in the visible range, while spirooxazine was less sensitive. Both compounds had a high absorbance for germicidal wavelength with a significant changed in color (as shown in Figure 2) which rendered them reliable germicidal UV indicators.

\subsection{Parameters Influencing the Deactivation of the Merocyanine Form}

After activation of both samples under $325 \mathrm{~nm}$ light, the effect of temperature on the merocyanine form of these compounds was investigated. For these tests, the time needed for spirooxazine and spiropyran to change back to their original colors (transparent and pale-yellow, respectively) after UV irradiation was recorded for different temperature. Spirooxazine and spiropyran with a concentration of $1 \%$ were used and it was observed that the temperature had a significant effect on the deactivation of the compounds as shown in (Figure 5). Indeed, for both compounds, the more the temperature increased, the faster was the deactivation, and spirooxazine exhibited a better response to temperature than spiropyran. Both of the curves show that the deactivation time was much longer for temperature below $60^{\circ}$. At $50^{\circ} \mathrm{C}$, the spirooxazine needed 6 minutes while spiropyran needed 25 minutes. At $70^{\circ} \mathrm{C}$, spirooxazine deactivated in less than one minutes and spiropyran in 5 minutes.

Above $80^{\circ} \mathrm{C}$, spirooxazine recovered in a few seconds (a little bit more than 3 minutes for the spiropyran). These results clearly showed the impact of temperature on the merocyanine form of both compounds. According to the spectrum showed in Figure 4(b), spiropyran exhibited an absorbance peak in the visible after $325 \mathrm{~nm}$ irradiation. Thus, the effect of visible light on the deactivation time was investigated. The first step was to activate both compounds (merocyanine form) using the 325 $\mathrm{nm}$ laser, same as the method used previously during the thermal experiment. Then, a green laser (532 nm) and a red laser diode $(650 \mathrm{~nm})$ were used to deactivate the compounds, making them returned to their original state (closed ring form). The absorbance spectra of both compounds at wavelengths mentioned above were recorded as a function of time. The wavelengths had been chosen because according to the spectrum recorded in Figure 4(b), spiropyran was sensitive at $\lambda=532 \mathrm{~nm}$ with an absorbance of 0.48 for concentration of $1 \%$ and 1.62 for concentration of $3 \%$, while at $\lambda=650 \mathrm{~nm}$ it was negligible (only at 0.08 and 0.29 for concentration of 1 and $3 \%$ respectively). The power $(1 \mathrm{~mW})$ of both lasers had also intentionally been chosen low to avoid warming up the material and induce thermal recovery.

From Figure 6(a), spiropyran exhibited a high absorbance at $532 \mathrm{~nm}$ (1.88 and 0.52 after 5 seconds for concentration respectively at $3 \%$ and $1 \%$ ), which slowly decreased with time to a value of 0.02 (after 8 minutes

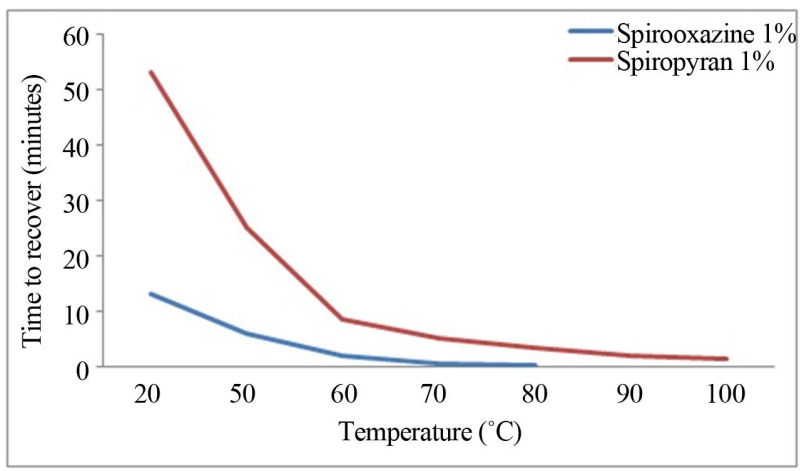

Figure 5. Effect of the temperature on of the deactivation of the merocyanine form of both compounds.
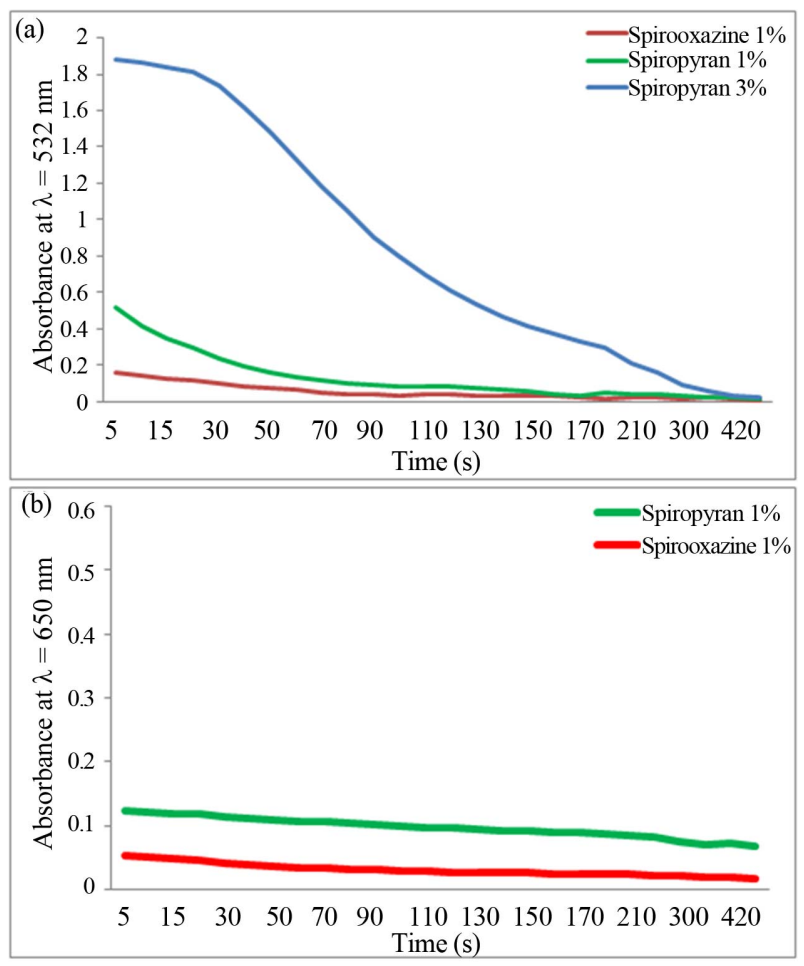

Figure 6. (a) Absorbance of spirooxazine and spiropyran under green laser vs time; (b) Absorbance of spirooxazine and spiropyran under red laser vs time. 
for both concentrations). This decrease was due to the fact that the colored merocyanine form of the spiropyran absorbed the green light and changed the chemical structure of the material back to the closed ring form. By simple inspection, it was observed a changing back in color from dark purple to pale yellow. Furthermore, the higher the concentration, the sensitive it was at this wavelength. For the spirooxazine, the absorbance was quite low ( 0.15 after 5 seconds) and dropped to less than 0.02 after 3 minutes. This was predicted from the absorbance spectrum (Figure 4(a)) recorded after UV irradiation. Note that, this material did not recover back to its original color and thus still remained blue at the end of the time set. The decreased in the absorbance could be due to a small thermal effect.

These results were compared with those obtained under red laser irradiation (Figure 6(b)), it was found that both compounds did not absorb this wavelength. After 5 seconds, the absorbance of spiropyran was at 0.12 , and that of spirooxazine was 0.05 . After 8 minutes, it was at 0.06 and 0.018 for spiropyan and spirooxazine respectively. Once again, the decrease was only due to small thermal effect which made both compound deactivated slowly from merocyanine form to closed ring form. At the end of the experiment with the red laser $(650 \mathrm{~nm})$, the predominant form of both materials was the merocyanine form (colored form). From the results shown in the experiments, the term "photochromism" employed to define spirooxazine should be used carefully as temperature was the dominant effect which induced the change of the merocyanine form into the closed ring form (from colored to colorless). As the deactivation was mainly thermally induced, the term thermochromism could also be used to define this material. For the spiropyran, we showed that only a certain wavelength band had an impact on the transformation of the merocyanine form, principally in the green. Therefore, the term "recover under visible light" $[1,22-26]$ can also be more specifically defined in terms of wavelength.

\subsection{Influence of $X$-Rays on the Spirooxazine and Spiropyran Closed Ring Form}

For completness, the materials were irradiated with XRay. The aim was to investigate if either photochromic compounds could be activated under X-Ray, and if their characteristic, such as photo fatigue resistance were affected. A change in color of both compounds could have led to new applications. Unfortunately, after X-Ray irradiation, both compounds did not change color. Therefore, other characteristics of the materials were tested with this irradiation, and it was found that this irradiation also did not influence the photofatigue resistance of spiropyran and spirooxazine.

\section{Conclusion}

A consistent photophysical characterization of 1,3-Dihydro-1,3,3-trimethylspiro[2H-indole-2,3'-[3H]naphth[2,1b] [1,4]oxazine] and 1',3'-Dihydro-1',3',3'-tri-methyl-6nitrospiro[2H-1-benzopyran-2,2'-(2H)-indole] compounds put into a PMMA matrix have been demonstrated by measuring their absorbance spectra. It was demonstrated that the temperature was the dominant effect in the deactivation of both compounds (after activation by $325 \mathrm{~nm}$ irradiation). It was found that spirooxazine was not sensitive to visible light after UV exposure, which contradicted results found in some articles. New results presented in this paper specified the main wavelengths for the deactivation of the spiropyran. The results showed that the term "thermochromism" could also be used to describe spirooxazine. We also showed that the materials were not affected by X-Rays which concluded the characterization.

\section{Acknowledgements}

We acknowledge support from the Hong Kong Polytechnic University's Institutional Research Fund under Project 4-BC06.

\section{REFERENCES}

[1] J. Żmija and M. J. Małachowski, "New Organic Photochromic Materials and Selected Applications," Journal of Achievements in Materials and Manufacturing Engineering, Vol. 41, No. 1, 2010, pp. 48-56.

[2] C. Bohne, M. G. Fan, Z. J. Li, Y. C. Liang, J. Lusztyk, and J. C. Scaiano, "Laser Photolysis Studies of Photochromic Processes in Spirooxazines: Solvent Effects on Photomerocyanine Behavior," Journal of Photochemistry and Photobiology A: Chemistry, Vol. 66, No. 1, 1992, pp. 79-90. http://dx.doi.org/10.1016/1010-6030(92)85121-A

[3] J. P. Boilot, J. Biteau, F. Chaput, T. Gacoin, A. Brun, B. Darracq, P. Georges and Y. Lévy, "Organic-Inorganic Solids by Sol-Gel Processing: Optical Applications," Pure and Applied Optics: Journal of the European Optical Society Part A, Vol. 7, No. 2, 1998, pp.169-177. http://dx.doi.org/10.1088/0963-9659/7/2/007

[4] A. K. Chibisov and H. Görner, "Photoprocesses in Spiropyran-Derived Merocyanines," The Journal of Physical Chemistry A, Vol. 101, No. 24, 1997, pp. 4305-4312. http://dx.doi.org/10.1021/jp9625691

[5] Y. Choi and Y. J. Choi, "The Effects of UV Disinfection on Drinking Water Quality in Distribution Systems," Water Research, Vol. 44, No. 1, 2010, pp. 115-122. http://dx.doi.org/10.1016/j.watres.2009.09.011

[6] C. A. Cotton, D. M. Owen, G. C. Cline and T. P. Brodeur, "UV Disinfection Costs: For Inactivating Cryptosporidium," Journal of American Water Works Association, Vol. 93, No. 6, 2001, pp. 82-94.

[7] J. C. Crano, W. S. Kwak and C. N. Welch, "Applied 
Photochromic Polymer Systems," Chapman and Hall Edition, New York, 1992.

[8] S. Cui, Y. Zhan, S. Pu and W. Liu, "Synthesis of New Photochromic Diarylethenes Bearing Five and Six Ring Aryls for Optical Storage," Symposium on Photonics and Optoelectronics (SOPO), Wuhan, 16-18 May 2011, pp. 1-4. http://dx.doi.org/10.1109/SOPO.2011.5780404

[9] C. Fitzpatrick, E. Lewis, A. Al-Shamma'a and J. Lucas, "An Optical Fibre Sensor for Germicidal Microwave Plasma Powered UV Lamps Output with Potential for On-Line Temperature Control," Optical Fiber Sensors Conference Technical Digest, Portland, 10 May 2002, pp. 455-458. http://dx.doi.org/10.1109/OFS.2002.1000690

[10] A. Gadgil, D. Greene, A. Drescher and S. Wiel, "Status Report on UV Waterworks: Water Disinfection for the Developing World," Lawrence Berkeley National Laboratory Report Number LBNL-40730, August 1997. http://energy.lbl.gov/iep/archive/uv/uv-status-rpt.html

[11] R. Guglielmetti, "Heterocyclo-Annulated Spirooxazines and 2H-Caromenes: Two Complementary Series of Photochromic Compounds," Molecular Crystals and Liquid Crystals Science and Technology, Vol. 298, No. 1, 1997, pp. 13-20. http://dx.doi.org/10.1080/10587259708036137

[12] Y. Huang, W. Liang, J. K. Poon, Y. Xu, R. K. Lee and A. Yariv, "Spiro-Oxazine Photochromic Fiber Optical Switch," Applied Physics Letters, Vol. 88, No. 18, 2006, pp. 181102-181103.

[13] S. Kawata, "Photorefractive Optics in Three-Dimensional Digital Memory," Proceedings of the IEEE, Vol. 87, No. 12, 1999, pp. 2009-2020. http://dx.doi.org/10.1109/5.805917

[14] K. Matsui and S. Yoshida, "Photochromic Film of 6-nitro-1',3',3'-trimethylspiro-[2H-1-benzopyran-2,2'-indolin e] Prepared by Plasma Polymerization," Journal of Applied Physics, Vol. 64, No. 5, 1988, pp. 2607-2610. http://dx.doi.org/10.1063/1.341650

[15] C. Salemi-Delvaux, B. Luccioni-Houze, G. Baillet, G. Giusti and R. Guglielmetti, "Effect of Photodegradation on the Thermal Bleaching Rate Constant of Photochromic Compounds in Spiro[Indoline-Pyran] and Spiro[IndolineOxazine] Series," Journal of Photochemistry and Photobiology A: Chemistry, Vol. 91, No. 3, 1995, pp. 223-232. http://dx.doi.org/10.1016/1010-6030(95)04113-X

[16] J. Mysliwiec, L. Sznitko, S. Bartkiewicz, A. Miniewicz, Z. Essaidi, F. Kajzar and B. Sahraoui, "Amplified Spontaneous Emission in the Spiropyran-Biopolymer Based System," Applied Physics Letters, Vol. 94, No. 24, , 2009, Article ID: 241106. http://dx.doi.org/10.1063/1.3155203
[17] D. Sridharan, E. Waks, J. T. Fourkas and G. Solomon, "Reversible Tuning of Photonic Crystal Cavities Using Photochromic Films," Conference on Lasers and Electro-Optics 2010, San Jose, 16-21 May 2010, pp. 1-2.

[18] S. Pissadakis, D. Anglos, A. Klini and M. Konstantaki, "Long Period Optical Fiber Grating Outcladding Overlaid Sensors: A Versatile Photonic Platform for Health and Bio Applications," 2011 International Workshop on Biophotonics, Parma, 8-10 June 2011, pp. 1-3.

[19] G. Liu, W. Liu and C. M. Dong, "UV- and NIR-Responsive Polymeric Nanomedicines for On-Demand Drug Delivery," Polymer Chemistry, Vol. 4, No. 12, 2013, pp. 3431-3443. http://dx.doi.org/10.1039/c3py21121e

[20] M. Li, Z. Qiang, J. R. Bolton and W. Ben, "Impact of Reflection on the Fluence Rate Distribution in a UV Reactor with Various Inner Walls as Measured Using a Micro-Fluorescent Silica Detector," Water Research, Vol. 46, No. 11, 2012, pp. 3595-3602. http://dx.doi.org/10.1016/j.watres.2012.04.004

[21] W. A. M. Hijnen, E. F. Beerendonk and G. J. Medema, "Inactivation Credit of UV Radiation for Viruses, Bacteria and Protozoan (Oo)cysts in Water: A Review," Water Research, Vol. 40, No. 1, 2006, pp. 3-22. http://dx.doi.org/10.1016/j.watres.2005.10.030

[22] L. J. Rose and H. O'Connell, "UV Light Inactivation of Bacterial Biothreat Agents," Applied and Environmental Microbiology, Vol. 75, No. 9, 2009, pp. 2987-2990. http://dx.doi.org/10.1128/AEM.02180-08

[23] M. Mcsherry, C. Fitzpatrick and E. Lewis, "An Optical Fiber Sensor for the Detection of Germicidal UV Irradiation Using Narrowband Luminescent Coatings," Sensor Journal, Vol. 4, No. 5, 2004, pp. 619-626. http://dx.doi.org/10.1109/JSEN.2004.833504

[24] B. Van Gemert, "Benzo and Naphthopyrans (Chromenes)," In: J. C. Crano and R. J. Guglielmetti, Eds., Organic Photochromic and Thermochromic Compounds, Springer, US, 2002, pp. 111-140.

[25] G. Ballet, "Photodegradation of Organic Photochromes in Polymers-Naphthopyrans and Naphthoxazines Series," Molecular Crystals and Liquid Crystals Science and Technology, Section A. Molecular Crystals and Liquid Crystals, Vol. 298, No. 1, 1997, pp. 75-82. http://dx.doi.org/10.1080/10587259708036145

[26] H. Hattori and T. Uryu, "Photochromic Chiral Liquid Crystalline Systems Containing Spiro-Oxazine with a Chiral Substituent II. Photoinduced Behaviour," Liquid Crystals, Vol. 28, No. 7, 2001, pp. 1099-1104. http://dx.doi.org/10.1080/02678290110048750 